\title{
Stage IVB Mycosis Fungoides and Sezary Syndrome AJCC v7
}

National Cancer Institute

\section{Source}

National Cancer Institute. Stage IVB Mycosis Fungoides and Sezary Syndrome A/CC v7. NCI Thesaurus. Code C88162.

Stage IVB includes: T1-4, N0-3, M1, B0-2. T1: Limited patches, papules, and/or plaques covering less than $10 \%$ of the skin surface. May further stratify into T1a (patch only) vs. T1b (plaque +/- patch). T2: Patches, papules, or plaques covering $10 \%$ or more of the skin surface. May further stratify into T2a (patch only) vs. T2b (plaque +/- patch). T3: One or more tumors (equal or greater than $1 \mathrm{~cm}$ diameter). T4: Confluence of erythema covering $80 \%$ or more of body surface area. N0: No clinically abnormal peripheral lymph nodes; biopsy not required. N1: Clinically abnormal peripheral lymph nodes; histopathology Dutch grade 1 or NCI LN0-2. N2: Clinically abnormal peripheral lymph nodes; histopathology Dutch grade 2 or NCI LN3. N3: Clinically abnormal peripheral lymph nodes; histopathology Dutch grades 3-4 or NCI LN4; clone positive or negative. M1: Visceral involvement (must have pathology confirmation and organ involved should be specified). B0: Absence of significant blood involvement: $5 \%$ or less of peripheral blood lymphocytes are atypical (Sezary cells). B1: Low blood tumor burden: more than 5\% of peripheral blood lymphocytes are atypical (Sezary cells) but does not meet the criteria of B2. B2: High blood tumor burden: 1000/microL Sezary cells or more with positive clone. (AJCC 7th ed.) 